\title{
Gyroscopic Magnetic Levitation: an Original Design Procedure Based on the Finite Element Method
}

\author{
Zacharie De Grève ${ }^{1,2 a}$, Christophe Versèle ${ }^{1}$, and Jacques Lobry ${ }^{1}$ \\ 1 UMons, Faculté Polytechnique de Mons, Service de Génie Electrique, Bd Dolez 31, B-7000 Mons \\ 2 F.R.S/FNRS, Fonds de la Recherche Scientifique, Rue d'Egmont 5, B-1000 Bruxelles
}

Received: date / Revised version: date

\begin{abstract}
In this work, an original procedure, based on the finite element method, is presented for the design of a Levitron ${ }^{(}$, a device made of permanent magnets and relying on stable gyroscopic magnetic levitation, using secondhand components. A perturbation force analysis is performed on finite element models of available magnets in order to derive the locus of stable equilibrium, as well as the top mass, for a given configuration of the magnets. We investigate three methods for the estimation of forces from finite element computations, two of them based on the virtual work principle, and one performing numerical integration of the classical expression of forces between magnets. Results are employed to realize a Levitron $^{\left({ }^{(}\right)}$in laboratory, and are shown to be in better agreement with experience than those from a simple analytical model available in the literature.
\end{abstract}

PACS. 85.70.Rp Magnetic levitation, propulsion and control devices - 02.70.Dh Finite-elements and Galerkin methods - 07.55.Db Generation of magnetic fields ; magnets

\section{Introduction}

Even if common people are more familiar with the attractive aspect of magnetic forces (lifting electromagnets, actuators, etc.), attention must be paid to magnetic repulsion forces, for the numerous possibilities they offer. For instance, when they are opposed to gravity in a carefully designed system, stable magnetic levitation can be made possible. Scientists and engineers, aware of the impact this phenomenon could have in every day life, have been particularly concerned with magnetic levitation these years (magnetic levitation trains, such as Japanese Maglev and German Transrapid, are good examples).

Earnshaw proved in 1842 that static fields are not able to maintain a magnetized body in stable levitation ${ }^{1}$. Consequently, in order to make magnetic levitation viable, researchers had to lean towards other possibilities. One solution is based on the use of superconductor materials. Indeed, these can be considered as perfect diamegnetic bodies $\left(\mu_{r} \simeq 0\right)$, which have the property to repel applied magnetic fields, and are not embraced in Earnshaw's theory [1,2]. The Japanese Maglev uses for instance the principle of superconductor levitation. Another way to avoid Earnshaw's theorem limitations is to include a dynamical

\footnotetext{
a e-mail : zacharie.degreve@umons.ac.be

1 Earnshaw's theorem precludes the existence of potential extrema for a static configuration of electric (or magnetic) particles, thus forbidding stable equilibrium.
}

aspect in the system. In some applications, such as the German Transrapid, a feedback loop regulates the current flowing in electromagnet windings, in order to continuously adjust the train motions. In a more academic solution, the magnetic levitation phenomenon is produced by Laplace forces acting on induced current loops, circulating in a conducting body placed in an alternating magnetic field.

In the 1990s, two systems were developed, for which a magnetized spinning top was maintained in stable levitation above a base magnet: the Japanese $\mathrm{U}_{-} \mathrm{CAS}{ }^{\circledR}$ and the American Levitron ${ }^{\circledR}$. In both cases, the levitation force is caused by magnetic repulsion forces between permanent magnets. However, the top of the U-CAS ${ }^{\complement}$ has a conductive coating on its surface, so that its horizontal stability is assured by the eddy currents flowing in it [3]. In the Levitron ${ }^{\circledR}$, the rotating magnet is maintained in stable levitation by gyroscopic effects. More precisely, the Levitron $^{\circledR}$ is made up of a top (a non-magnetic spindle inserted in a flat, or toroïdally shaped, permanent magnet) and of a magnetized base with a circular hole on its centre. The gyroscopic torques acting on the spinning top maintain it in a nearly vertical alignment, so as to prevent it being flipped over. But stability against flipping is not enough: the phenomenon can not be explained if the top's axis has a fixed direction in space. Gyroscopic precession is needed, so that the top's axis is continuously aligned to the base magnetic field direction [4-6]. An unmagnetized 


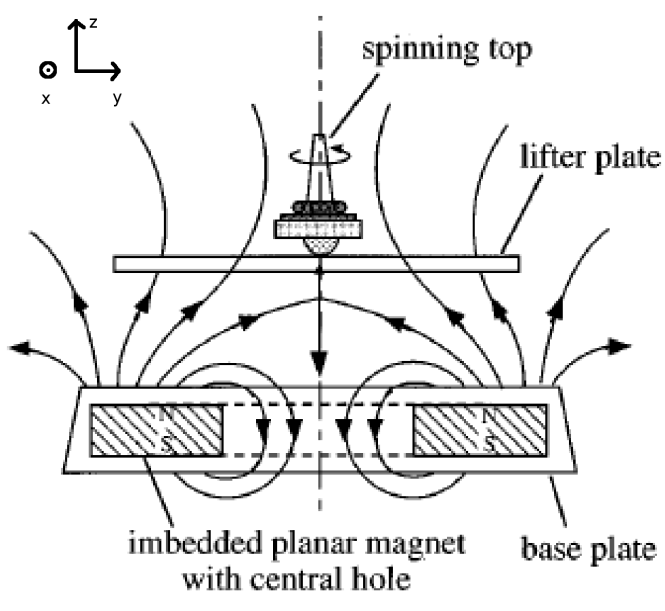

Fig. 1. The Levitron ${ }^{\circledR}[5]$.

guide is also required in order to bring the rotating top into the area of stable equilibrium (Fig. 1).

This work aims to design and realize a Levitron ${ }^{(c)}$ in laboratory, using secondhand components such as ferrite permanent magnets from old speakers and NeodymiumIron-Bore magnets. To do so, the magnetic component parameters are first identified (in terms of volume magnetization $\boldsymbol{m}$ ). This is done by comparing magnetic induction measurements along the $z$ axis of the magnet, obtained via a Hall effect gaussmeter, and the induction estimated by means of finite element models (see sections 2 and 3 ). Then, a perturbation force analysis is applied to derive the locus of stable equilibrium: stability is obtained when top radial or axial excursions are compensated by opposite perturbation forces (see section 4). The top mass is thereafter estimated by opposing the magnetic force exerted on it to gravity. Three methods will be compared for the computation of magnetic forces, two based on the virtual work theory and one performing the numerical integration of the classical expression of forces between permanent magnets (subsection 4.3). Finally, after being compared with a simple analytical model available in the literature [5] in which the top is considered as a pointwise magnetic dipole, the results will be employed to create a Levitron ${ }^{\circledR}$, by assembling the identified components. It will be shown that our approach conducts to results in better agreement with the experience than those obtained elsewhere [7].

It is important to note that this paper focuses on magnetic aspects only, in order to derive the conditions assuring stable levitation. Mechanical aspects are not addressed in this study: the impact of top rotation speed against stability is for instance not discussed. However, the authors are convinced that this approach can be used as a base for the comprehension of the complex phenomenology of the Levitron $\left.{ }^{(}\right)$, and suggest references $[4,6,8,9]$ for more detailed models.

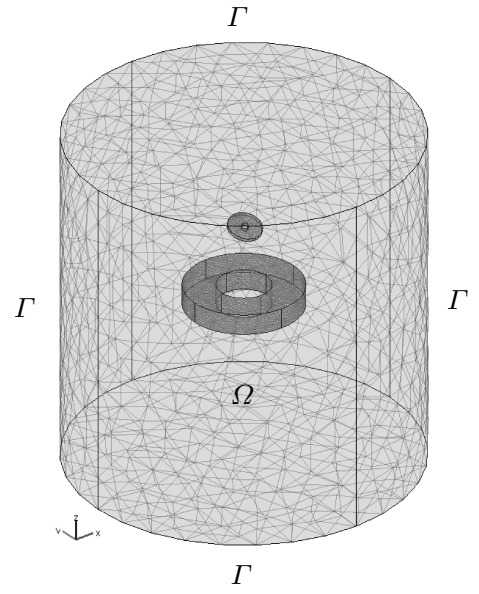

Fig. 2. Three-dimensional model of our Levitron ${ }^{\circledR}$.

\section{Numerical model}

Due to the absence of real currents, the total magnetic scalar potential $\psi$, defined as follows, is employed:

$$
\boldsymbol{h}=-\operatorname{grad} \psi
$$

where $\boldsymbol{h}$ stands for the magnetic field. The magnets' constitutive law is the following, with $\boldsymbol{b}$ the magnetic induction, $\mu_{0}$ the vacuum permeability and $\boldsymbol{m}$ the volume magnetization, constant and oriented along the $z$ axis (rigid permanent magnets):

$$
\boldsymbol{b}=\mu_{0}(\boldsymbol{m}+\boldsymbol{h})
$$

Combining equations (1) and (2) with the Gauss law of magnetism yields the local form to be solved for $\psi$ on the whole domain $\Omega$ (which is constituted by the magnets and a surrounding cylinder-shaped air box, see Fig. 2):

$$
\begin{array}{cccc}
\Omega: & -\mu_{0} \operatorname{div}(\operatorname{grad} \psi)+\mu_{0} \boldsymbol{m} & =\mathbf{0} \\
\Gamma: & \partial_{n} \psi & =0
\end{array}
$$

Equation (4) completes the model by specifying conditions to be fulfilled on the boundary $\Gamma$ of domain $\Omega$ (magnetic field purely tangential along system boundaries). We decomposed the problem geometry into tetrahedra, and approximated $\psi$ on each element using second-order Lagrangian nodal basis functions $N(x, y, z)$ :

$$
\hat{\psi}=\sum_{i=1}^{10} N_{i}(x, y, z) \hat{\psi}_{i}
$$

In equation (5), $\hat{\psi}$ stands for the approximated total scalar magnetic potential, and $\hat{\psi}_{i}$ for the values of $\hat{\psi}$ on the ten nodes of the second-order tetrahedron. A weak form of problem (3-4) is then derived using the Galerkin method [10], and $\hat{\psi}$ is introduced in the obtained equations, leading to a linear system to be solved for the $\hat{\psi}_{i}$. A Conjugate Gradient resolution procedure shows fast convergence towards the solution. 


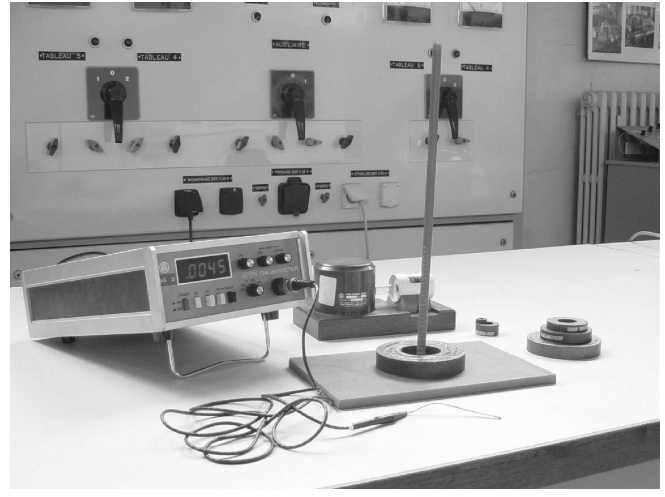

Fig. 3. Our measurement station (model 912 gaussmeter $R F L$ Industries Inc.).

Radial excursions of the top, as it will be explained in section 4.2 , led us to use a $3 D$ cartesian model rather than a simpler $2 D$ axisymmetric one (see Fig. 2).

\section{Magnetic component identification}

As no information is a priori available for the ferrite and Neodymium-Iron-Bore magnets, an identification phase is first needed, in order to derive accurate magnetic models for the components. To that end, magnetic induction measurements along the $z$-axis of the magnet, obtained using a Hall effect gaussmeter (see Fig. 3), are compared with the induction evaluated by means of finite element models. An estimator, the SNSE (for Sum of Normalized Squared Errors), is computed to account for the curves adjustment quality:

$$
S N S E=\sum_{i=1}^{n}\left(\frac{h_{i, m e s}-h_{i, s i m}}{h_{i, m e s}}\right)^{2}
$$

The model volume magnetization $\boldsymbol{m}=m \boldsymbol{u}_{\boldsymbol{z}}$ is tuned in order to minimize the SNSE. Fig. 4 compares the measured and simulated magnetic fields, for magnet $B_{5}$, and table 1 summarizes the identification results, as well as the magnet dimensions (outer and inner diameters, respectively $d_{o}$ and $d_{i}$, and magnet height $e$ ). Note that the $B_{i}$ magnets are candidate for the Levitron ${ }^{(C)}$ base construction whereas the $T_{i}$ ones are for the top. Worse SNSEs are obtained for the top magnets, as these are subject to greater measurement errors considering their small size: an accurate experimental characterization of the $h$ curves is indeed trickier as the magnet dimensions decrease, especially in the positive slope area.

\section{Design procedure}

\subsection{Static equilibrium}

Static equilibrium is obtained when gravity is compensated by the magnetic force exerted on the top. If $M$

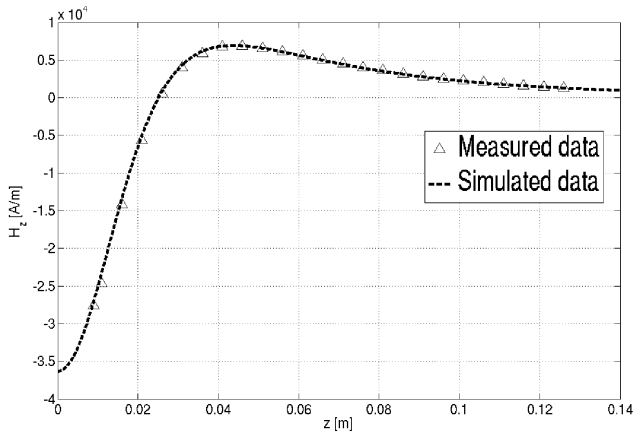

Fig. 4. Identification of magnet $B_{5}$. This figure shows the magnetic field along the magnet $z$ axis, obtained experimentally and numerically, versus the distance from the magnet centres.

Table 1. Results of the magnet identification phase. All the magnets are made up of ferrite, except $T_{3}$ which is made up of Nd-Fe-B.

\begin{tabular}{llllll}
\hline Code & $d_{o}[\mathrm{~mm}]$ & $d_{i}[\mathrm{~mm}]$ & $e[\mathrm{~mm}]$ & $m[\mathrm{~A} / \mathrm{m}]$ & $S N S E$ \\
\hline$B_{1}$ & 94 & 44 & 16 & 183000 & 0.078 \\
$B_{2}$ & 82 & 38 & 15 & 172500 & 0.024 \\
$B_{3}$ & 64 & 24 & 12 & 227500 & 0.024 \\
$B_{4}$ & 52 & 22 & 9 & 250000 & 0.076 \\
$B_{5}$ & 101 & 46 & 18 & 192000 & 0.038 \\
$T_{1}$ & 36 & 18.5 & 6 & 190000 & 0.22 \\
$T_{2}$ & 32.5 & 16 & 8 & 260000 & 0.021 \\
$T_{3}$ & 29 & 6 & 3 & 765000 & 0.1 \\
\hline
\end{tabular}

stands for the top mass, $\boldsymbol{g}$ for the terrestrial gravitational acceleration and $f_{m, z}$ for the $z$ component of the magnetic force $\boldsymbol{f}_{\boldsymbol{m}}$, we have:

$$
\boldsymbol{f}_{\boldsymbol{m}}=f_{m, z} \boldsymbol{u}_{\boldsymbol{z}}=M \boldsymbol{g}
$$

\subsection{Stability analysis}

For stable equilibrium to exist, small displacements of the top in any direction should be compensated by opposite forces, which would put it back in its previous position. In other words, force field lines should all point inwards, towards the equilibrium position, which means that the divergence of the force field should be negative. However, Earnshaw's theorem states that such a situation cannot be encountered with static magnetic fields. In the Levitron ${ }^{\circledR}$, the spinning top acts as a gyroscope, preventing its magnetic field to align itself in the same direction as that of the base. This flipping phenomenon, combined with the top precession and nutation motions, allow the existence of a stable equilibrium area, where gravitation, magnetic and gyroscopic forces are compensated [5]. In our approach, based on [5], these two motions (precession and nutation) are ignored (orientational stability is considered as given), whereas assumptions are made about the top orientation during excursions around the equilibrium position. More sophisticated models, which account 
for the complex dynamics of the Levitron ${ }^{\circledR}$, are available in the literature $[4,6,8,9]$.

Two models are investigated in this work [5]. The first one assumes that the top is spinning so rapidly that gyroscopic action maintains its magnetic moment perfectly aligned with the $z$ axis, irrespective of radial or axial excursions (model $\left.M_{1}\right)$ :

$$
\boldsymbol{m}=m_{z} \boldsymbol{u}_{\boldsymbol{z}} \quad\left(\text { model } M_{1}\right)
$$

Magnetization norm is supposed to be constant (rigid permanent magnets case). The second model, $M_{2}$, assumes that the top remains parallel to the base magnetic field during radial or axial excursions around equilibrium, a phenomenon well observed in practice:

$$
\boldsymbol{m}=m \frac{\boldsymbol{h}}{\|\boldsymbol{h}\|} \quad\left(\text { model } M_{2}\right)
$$

The geometry configuration during radial excursions requires a three-dimensional model instead of a simpler axisymmetric one, as depicted in Fig. 2.

Basing on these considerations, our approach for the design of the Levitron $\left.{ }^{(}\right)$is the following. Axial (i.e. $z$ oriented) and radial perturbation forces acting on the top are computed from finite element simulations, for both models (equations (8) and (9)) and for different positions of the top along the $z$ axis. An equilibrium area is then derived, considering the fact that stability is assured when the perturbations are compensated (i.e. when the perturbation force is opposite to the displacement direction). Then, the top mass is estimated by opposing the magnetic force exerted on the top in the stability area to gravity (equation (7)).

\subsection{Force computation}

Three methods are investigated for the computation of perturbation forces from finite element simulations. One of them (NUMINT) performs a numerical integration of the classical expression of forces between magnets, whereas the two others are based on the virtual work principle (CVW and LVW). One might wonder why Maxwell stress tensor (MST) methods are not addressed in our work. In fact, in a finite element context, such methods imply the choice of a surface of integration in the air surrounding the cible body. For 3D cases, the influence of that choice on results may become non trivial [11]. On the other hand, virtual work based methods only require well-defined volume integrations as explained below, while keeping the same advantages as MST methods.

\subsubsection{Numerical integration}

The force exerted on a permanent magnet of magnetization $\boldsymbol{m}$ placed in an external magnetic field $\boldsymbol{h}$ (i.e. the base magnetic field) is given by [12]:

$$
\boldsymbol{f}_{\boldsymbol{m}}=\mu_{0} \iiint_{\Omega}(\boldsymbol{m} \operatorname{grad}) \boldsymbol{h} d \Omega
$$

Projecting equation (10) along the reference axis system yields :

$$
\begin{aligned}
f_{m, x} & =\mu_{0} \iiint_{\Omega}\left(m_{x} \partial_{x} h_{x}+m_{y} \partial_{y} h_{x}+m_{z} \partial_{z} h_{x}\right) d \Omega \\
f_{m, y} & =\mu_{0} \iiint_{\Omega}\left(m_{x} \partial_{x} h_{y}+m_{y} \partial_{y} h_{y}+m_{z} \partial_{z} h_{y}\right) d \Omega \\
f_{m, z} & =\mu_{0} \iiint_{\Omega}\left(m_{x} \partial_{x} h_{z}+m_{y} \partial_{y} h_{z}+m_{z} \partial_{z} h_{z}\right) d \Omega
\end{aligned}
$$

The magnetic field $\boldsymbol{h}$ is obtained from finite element solutions using equation (1). The integrals in equations (11) are computed by performing a second order Gauss quadrature on the top subdomain. This method will be refered as NUMINT throughout this paper.

\subsubsection{Coulomb Virtual Work method}

In [11], the virtual work principle is employed to derive an expression of the magnetic force exerted on a rigid body, using the local jacobian derivative method. For a $\boldsymbol{h}$ oriented formulation, the magnetic co-energy is differentiated along the virtual displacement $i$, keeping the nodal values of the scalar magnetic potential $\hat{\psi}_{i}$ constants, so as to obtain, for the force component along $i$ axis:

$$
\begin{gathered}
f_{m, i}=\sum_{e}\left(\iiint_{\Omega_{e}}-\boldsymbol{b} G^{-1} \partial_{i} G \boldsymbol{h} d \Omega_{e}+\right. \\
\left.\iiint_{\Omega_{e}}\left(\int_{0}^{b} b d h\right)\|G\|^{-1} \partial_{i}\|G\| d \Omega_{e}\right)
\end{gathered}
$$

In equation (12), the sum extends to all the elements $e$ of the model, $i$ stands for the direction of the virtual displacement and $\Omega_{e}$ represents the volume of the considered element. $G$ is the jacobian matrix of the transformation which maps global coordinates to local element coordinates. All the elements belonging to the body are displaced all together along $i$ direction. Three categories of elements appear: the fixed, the entirely movable and the distorted ones (see Fig. 5). It can be shown that the co-energy is only modified by the virtual displacement in elements belonging to the third category, i.e. in air elements surrounding the movable body (top magnet), so that equation (12) needs only to be computed on these elements. The amount of distorted elements can be arbitrarily fixed, increasing the number of available algorithms, but we chose the tetrahedron layer directly surrounding the top subdomain for simplicity. In that case, equation (12) becomes: 


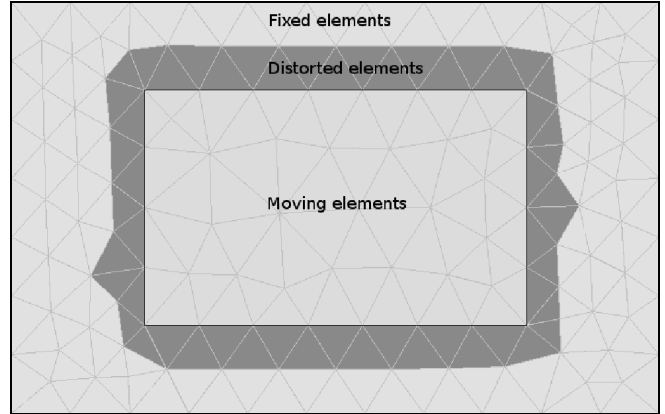

Fig. 5. Coulomb Virtual Work method. Equation (12) is only computed on dark elements.

$$
\begin{array}{r}
f_{m, i}=\sum_{e}\left(\iiint_{\Omega_{e}}-\mu_{0} \boldsymbol{h} G^{-1} \partial_{i} G \boldsymbol{h} d \Omega_{e}+\right. \\
\left.\iiint_{\Omega_{e}} \frac{\mu_{0}\|\boldsymbol{h}\|^{2}}{2}\|G\|^{-1} \partial_{i}\|G\| d \Omega_{e}\right),
\end{array}
$$

This procedure will be refered from now as CVW (for Coulomb Virtual Work method).

\subsubsection{Local Virtual Work method}

Unlike the CVW approach, where a set of nodes is simultaneously displaced, the local virtual work method (LVW) displaces a single node at a time $[13,14]$. Only the coenergy (for a $\boldsymbol{h}$ oriented formulation) corresponding to the elements surrounding that node is modified during the virtual displacement. Thus, a local force, associated to the node, can be obtained by differentiating the co-energy versus the virtual displacement at constant scalar magnetic potential. De Medeiros et.al. derived the force expression in the case of rigid permanent magnets [13] :

$$
\begin{array}{r}
f_{m, i, k}=\frac{\mu_{0}}{2} \sum_{e_{k}} \iiint_{\Omega_{e_{k}}}\left(-G^{-1} \partial_{i} G \boldsymbol{h}(\boldsymbol{h}+\boldsymbol{m})+\right. \\
(\boldsymbol{h}+\boldsymbol{m})\left(-G^{-1} \partial_{i} G \boldsymbol{h}\right)+(\boldsymbol{h}+\boldsymbol{m}) \\
\left.(\boldsymbol{h}+\boldsymbol{m})\|G\|^{-1} \partial_{i}\|G\|\right) d \Omega_{e_{k}}
\end{array}
$$

Terms in equation (14) have the same signification than in equation (13): $e_{k}$ stands for the elements surrounding node $k$, and the global force is obtained by summing the nodal forces on the nodes of the magnet. This method is a heuristic (all nodes are not displaced 'en bloc'), but has the advantage to give a repartition of the forces inside the magnet.

\section{Results and discussion}

Numerous combinations of base candidate and top candidate magnets were possible and investigated, but only

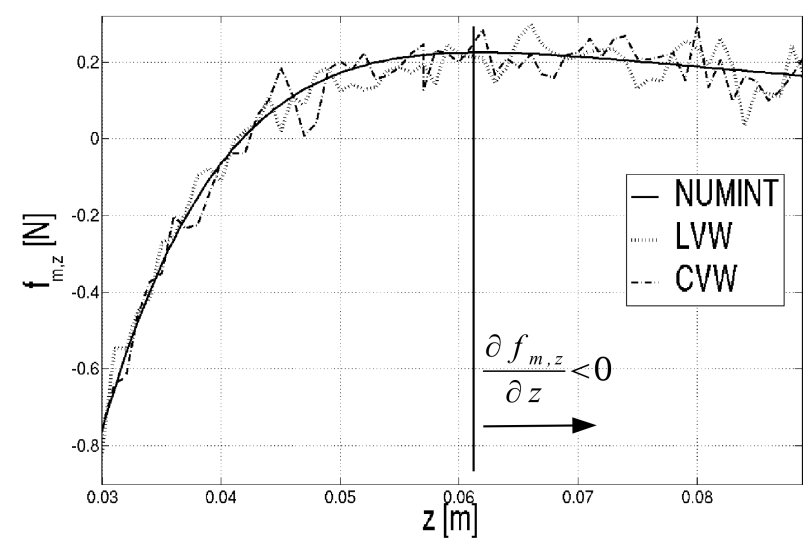

Fig. 6. Force exerted on the top ( $z$ component), for different positions of the base magnet along the $z$ axis, computed with the three methods NUMINT, CVW and LVW.

the results for the $B_{5}-T_{3}$ configuration will be exposed, as it is the authors' final choice. In that case, it took approximately $15 \mathrm{~s}$ to get one 3D mesh of nearly 150000 elements, using a $1.8 \mathrm{GHz}$ dual core processor with $4 \mathrm{~GB}$ RAM. We have adopted deliberately such a dense mesh in our example since accuracy, more than rapidity, was an issue. Solving the finite element model took more or less $40 \mathrm{~s}$, and the post-processing operations, i.e. radial and axial forces computation, took a few seconds, for the three methods. That procedure has been repeated for each position of the top along the $z$ axis, spaced by $0.0005 \mathrm{~mm}$, thus leading to a total simulation time of approximately 50 min for the CVW and LVW methods, and 20 min for the NUMINT method. Indeed, in the latter case, there is no need for solving the finite element model at each step since the magnetic field involved in equation (10) is the base magnetic field only.

Figure 6 shows the evolution of the $z$ component of the magnetic force acting on the top as the distance between the two magnets changes. We are only interested in the piece of curve with a negative slope, as it corresponds to an area where axial perturbations (i.e. $z$ oriented) are compensated. Forces obtained with the three methods (NUMINT, CVW and LVW) are represented. We observe that, considering the small value of the total force in our configuration (around $0.2 \mathrm{~N}$ ), CVW and LVW methods suffer from an excessive sensibility to the top position and meshing. For that reason, the following results will be exposed for the NUMINT method only. It is worth noting that a classical meshing algorithm (based on the Delaunay triangulation) was employed in order to automatically remesh the entirety of our system at each step (namely at each position of the top). We just forced the number of elements constituting the magnet to belong to a specific interval (1520 - 1540 tetrahedra). This does not mean that the element layer surrounding the top magnet keeps the same configuration at each step. As the virtual work based 


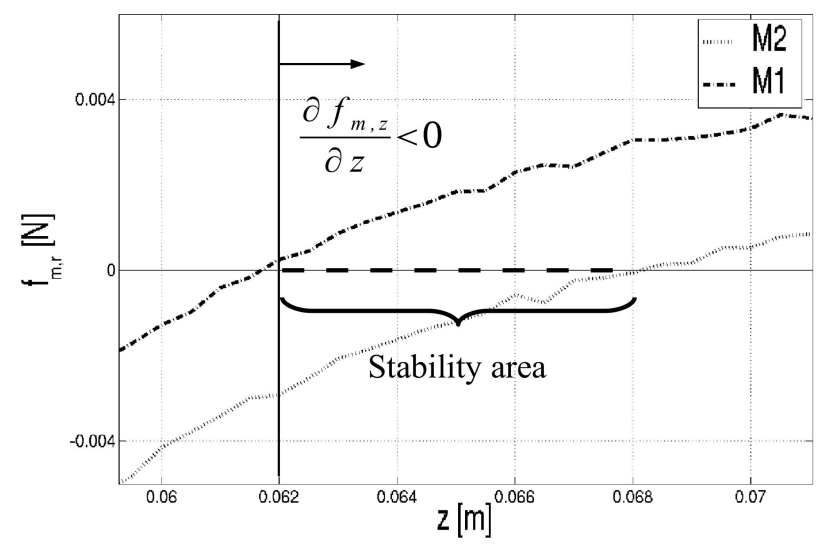

Fig. 7. Radial perturbation forces during positive top radial excursions, for the two $m$ oriented models (NUMINT method). Perturbations are compensated when force and displacement have opposite signs.

methods only require a numerical integration on this type of elements (see subsections 4.3.2 and 4.3.3), we understand the numerical noise which appears on Fig. 6. On the contrary, the NUMINT method requires an integration on the whole top, and is thus less influenced by the changing mesh. Further investigations would give a better understanding of the sensibility of the virtual work based methods for the present problem. For instance, we could use more sophisticated remeshing techniques, which for instance would keep exactly the same mesh for the rigid bodies at each step, and would deform the surrounding air elements using dedicated algorithms (see [15] for an overview of such methods). A combination of the finite element method with a boundary element method may also be considered. By doing so, the meshing of the air could be avoided, as well as the remeshing at each top position. For our purpose however, the NUMINT method gave satisfactory results.

In addition, in order to meet stability, radial perturbation forces have to be compensated when the top performs radial excursions from the $z$ axis. Figure 7 accounts for these forces, for the two models exposed in section 4.2, i.e. when $\boldsymbol{m}$ is rigidly oriented along $z\left(M_{1}\right)$ or when it is directed along the base magnetic field $\left(M_{2}\right)$. One can observe that only the second model gives satisfactory results: an area along the $z$ axis where axial and radial perturbation forces are simultaneously compensated can be found, whereas it is not possible for the first model. This is not surprising, since the second model is far more close to what is experimentally observed with a Levitron ${ }^{\circledR}$. To a certain extent, the approach dicussed in this paper gives a finite element validation of the assumptions about the orientation of $\boldsymbol{m}$ made in [5]. Moreover, it is important to note that the inconsistency of model $M_{1}$ demonstrates the existence of an upper limit for the top rotation speed, beyond which stability is not possible any more. Stable equilibrium is obtained for $z$ values in a range of $62 \mathrm{~mm}$

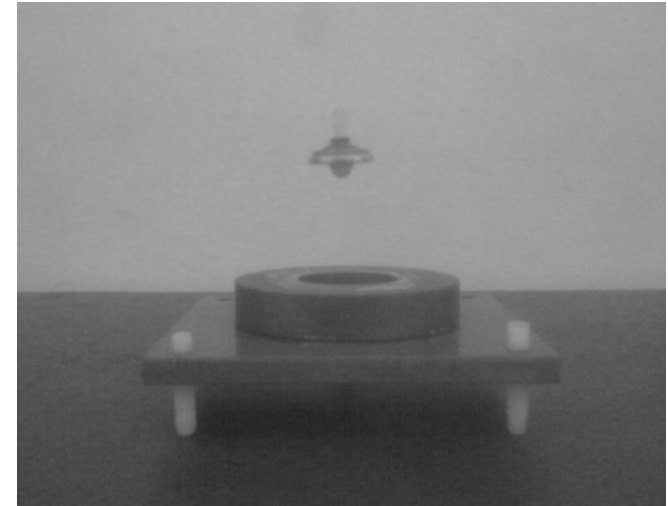

Fig. 8. Our Levitron ${ }^{\circledR}$

to $68 \mathrm{~mm}$. The lower limit corresponds to a top mass value of $22.8 \mathrm{~g}$ and the upper one to $22.3 \mathrm{~g}$.

These observations have been employed to realize a Levitron in laboratory. For the $B_{5}-T_{3}$ configuration, stability has been observed between $62 \mathrm{~mm}$ and $68 \mathrm{~mm}$ (as derived from the simulations), for a top weighing between $25.9 \mathrm{~g}$ and $26.2 \mathrm{~g}$. The gap between simulated and measured data can be partly explained by measurement errors. Indeed, the magnetic induction along the $z$ axis of the magnets has been measured with a gaussmeter, using a probe manipulated by hand, thus leading to inevitable approximations. When favorable conditions were gathered (base magnet carefully aligned with the vertical, external magnetic perturbations minimized, etc.), stable magnetic levitation has been observed during up to 1 min $22 \mathrm{~s}$ (Fig. 8).

Our results can be compared with a simple analytical model avalaible in the literature [5], in which the top is considered as a pointwise magnetic dipole. In [7], the authors computed a locus of stable equilibrium from $61 \mathrm{~mm}$ to $66 \mathrm{~mm}$ for the same magnet configuration, corresponding to top masses of $19.7 \mathrm{~g}$ and $20.3 \mathrm{~g}$. All the results are summarized in table 2 . We can observe that even if the stability areas coincide for the two approaches, the top mass obtained with the proposed method is in better agreement with the experimental measurements. This can be easily understood since the magnetic forces are here computed by numerically integrating over the entire top, instead of considering a simple dipole repulsion model.

\section{Conclusion}

In this paper, a finite element based procedure for the design of a Levitron ${ }^{\circledR}$, using secondhand components, has been presented. After identifying the magnets, a perturbation analysis has been performed in order to derive the locus of stable equilibrium, as well as the top mass, for various magnet combinations. It has been demonstrated that 
Table 2. Simulated and experimental results, for the $B_{5}-T_{3}$ configuration $\left(M_{2}\right)$

\begin{tabular}{llc}
\hline & Stability Area $[\mathrm{mm}]$ & Top mass $[\mathrm{g}]$ \\
\hline Our approach & $62-68$ & $22.3-22.8$ \\
$\begin{array}{l}\text { Magnetic dipole } \\
\text { approach }[5,7]\end{array}$ & $61-66$ & $19.7-20.3$ \\
$\begin{array}{l}\text { Experimental } \\
\text { results }\end{array}$ & $62-68$ & $25.9-26.2$ \\
\hline
\end{tabular}

the top flipping motion is required for stability: its magnetization vector must align itself with the base magnetic field during radial excursions, otherwise axial and radial perturbations cannot be simultaneously compensated. We have also emphasized that the virtual work based methods for the force computations, whereas giving satisfactory results in other general cases, suffer from an excessive sensibility to the top position and meshing, considering the small value of forces involved in our study. In our opinion, this effect should be reduced by implementing sophisticated meshing algorithms which take motion into account [15], instead of simply remeshing the entire system at each step, through a Delaunay triangulation. A combination of the finite element method with a boundary element method may also be considered, so as to avoid the meshing of the air as well as an entire remeshing at each top position. For our purpose, the numerical integration of the classical expression of forces exerted on permanent magnets has been successfully employed.

To a certain extent, the approach discussed in this article can be viewed as a finite element extension of [5], in which the top is assimilated to a pointwise magnetic dipole, and in which a simple analytical model is derived. Stability areas have been proven to be in good agreement with the experience for the two models. However, the top mass is better estimated with our method, which can be easily explained as forces are computed by integrating over the entire top geometry rather than considering simple dipole repulsion forces.

\section{References}

1. M.V. Berry and A.K. Geim, Eur. J. Phys. 18 (1997), 307313

2. E.H. Brandt, Phys. World 10 (1997), 23-24

3. M. Tsuchimoto, IEEE. Trans. on Mag. 35 (1999), no. 3, 1270-1273

4. M.D. Simon, L.O. Heflinger and S.L. Ridgway, Am. J. Phys. 65 (1997), no. 4, 286-292

5. T.B. Jones, M. Washizu and R. Gans, J. Appl. Phys. 82 (1997), no. 2, 883-888

6. M.V. Berry, Proc. R. Soc. Lond. A452 (1996), 1207-1220

7. Z. De Grève, C. Versèle and J. Lobry, J3eA 8 (2009), Hors série 1,1012
8. R.F. Gans, T.B. Jones and M. Washizu, J. Appl. Phys. 31 (1998), 671-679

9. S. Gov, S. Shtrikman and H. Thomas, Physica. 126 (1998), no. $3-4,214-224$

10. O. Zienkiewicz, La méthode des éléments finis (McGrawHill, Paris 1979), pp 55-64

11. J.L. Coulomb, IEEE Trans. on Mag. 19 (1983), no. 6, 25142519

12. E. Durand, Magnétostatique (Masson et Cie 1968)

13. L.H. De Medeiros, G. Reyne, G. Maunier and J.P. Yonnet, IEEE Trans. on Mag. 34 (1998), no. 5, pp 3012-3015

14. A. Benhama, A.C. Williamson and A.B.J. Reece, IEE Proc.-Electr.Power Appl. 147 (2000), no. 6, pp 437-442

15. V. Leconte, Electromagnétisme et éléments finis, edited by G. Meunier (Hermes Sciences, Lavoisier 2002), 3, pp 88-104 